\title{
The Difficulty of Easy Projects
}

\author{
By Wioletta Dziuda, A. Arda Gitmez, and Mehdi Shadmehr*
}

We consider binary private contributions to public good projects that succeed when the number of contributors exceeds a threshold. We show that for standard distributions of contribution costs, valuable threshold public good projects are more likely to succeed when they require more contributors. Raising the success threshold reduces free-riding incentives, and this strategic effect dominates the direct effect. Common intuition that easier projects are more likely to succeed only holds for cost distributions with right tails fatter than Cauchy. Our results suggest government grants can reduce the likelihood that valuable threshold public good projects succeed. (JEL D71, H41, H81)

Common sense suggests that a regime that collapses if at least 10 percent of its citizens revolt is more fragile than a regime that collapses if at least 20 percent of its citizens revolt. It is also natural to think that, all else equal, public good projects that require the contribution of a larger fraction of the population (e.g., referendums with higher passage thresholds) are always less likely to succeed. We show this intuition is wrong for high-value threshold public goods with binary contributions: among public good projects with high and identical values, those that require more participation for success are also more likely to succeed. For example, weakening the regime, lowering the passage threshold, or providing government grants can paradoxically reduce the likelihood of success in such projects.

We consider the classical threshold public good framework of Palfrey and Rosenthal (1984, 1988)(Fudenberg and Tirole 1991), in which the public good is provided whenever a sufficient number of citizens contribute. Figure 1 represents the game. Here, $N \geq 2$ citizens must simultaneously decide whether to contribute to a public good project. The payoff of the status quo is normalized to 0 . A project succeeds if at least $q$ citizens contribute, where $1 \leq q \leq N$. If the project succeeds, each citizen receives a payoff $u>0$. If citizen $i$ contributes, she pays a cost $c_{i}$. We assume that $c_{i}$ is citizen $i$ 's private information and that $c_{i} \sim_{i i d} F$ with $F(0) \in(0,1)$. We allow for $c_{i}$ to be negative to capture citizens who contribute regardless of what

\footnotetext{
*Dziuda: Harris School of Public Policy, University of Chicago (email: wdziuda@uchicago.edu); Gitmez: Department of Economics, Bilkent University (email: arda.gitmez@bilkent.edu.tr); Shadmehr: Department of Public Policy, University of North Carolina at Chapel Hill (email: mshadmeh@gmail.com). Larry Samuelson was coeditor for this article. We are grateful for the comments of Daron Acemoglu, Nageeb Ali, Scott Ashworth, Dan Bernhardt, Chris Berry, Raphael Boleslavsky, Peter Buisseret, Georgy Egorov, Alireza Tahbaz-Salehi, and James Robinson.

${ }^{\dagger}$ Go to https://doi.org/10.1257/aeri.20200311 to visit the article page for additional materials and author disclosure statement(s).
} 


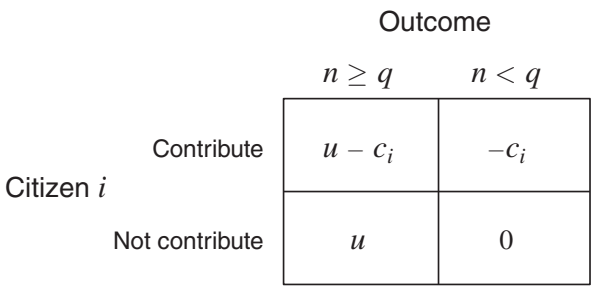

FIGURE 1

Notes: Threshold public good game with success threshold $q$. A public good with value $u$ is provided if the number of citizens who contribute, $n$, exceeds the threshold $q$.

others do and the success of the project, which allows us to rule out the equilibrium in which no one contributes. To ease exposition, we assume that $F(c)$ is differentiable on its support.

This framework captures the essence of various public good problems and has been adopted extensively in the literature. For example, "if 10 percent of the registered voters sign a petition at the city hall, a tax limitation referendum is held," or, "50 people see an assault taking place. If one person calls the police, the victim is saved" (Palfrey and Rosenthal 1984, p.172). Other examples include voting in committees (Taylor and Ward 1982), passing the budget in legislature (Myatt and Wallace 2008), legislators voting to raise their own pay (Ordeshook 1986), participation in meetings (Osborne, Rosenthal, and Turner 2000), boycotts (Diermeier and Van Mieghem 2008), tender offers (Palfrey and Rosenthal 1988), vaccination (Sandler 2015), and lobbying (Hansen 1985, Marwell and Oliver 1991) as well as the ratification of international treaties (Saijo and Yamato 1999) and joining an alliance in a world war (Goeree and Holt 2005). The large experimental literature that uses this framework includes Dawes et al. (1986); Offerman, Sonnemans, and Schram (1996); Cadsby and Maynes (1999); Spiller and Bolle (2017); and Palfrey, Rosenthal, and Roy (2017).

Our main result compares analytically the likelihood of public good provision for different success thresholds when the value of public good $u$ is sufficiently small or sufficiently large (Theorem 1). We prove that when the value of public good $u$ is small, the likelihood that the project succeeds is decreasing in the threshold $q$. This is consistent with the common sense that easier projects are more likely to succeed. In sharp contrast, we prove that when the value of public good $u$ is sufficiently large, the likelihood that the project succeeds is increasing in the threshold $q$ for cost distributions with bounded support or with an asymptotically increasing hazard rate. ${ }^{1}$ We provide simulations to glean insight about how the likelihood of success varies with the success threshold for intermediate public good values.

The direct effect of raising the success threshold $q$ reduces the likelihood of success: for the same citizens' behavior, it is less likely that at least $q+1$ of them contribute than that at least $q$ of them contribute. But there is also an indirect effect. A higher success threshold changes the probability that a citizen is pivotal and hence

\footnotetext{
${ }^{1}$ That is, when $1-F(c)$ is log-concave for sufficiently large $c$. If $f(c)$ is log-concave, so is $1-F(c)$.
} 
her incentives to contribute. When the public good value $u$ is large, a higher success threshold raises the probability of pivotality and hence contribution incentives. We characterize when this indirect effect dominates.

To see that the indirect effect can dominate, consider the special case with unanimity and bounded support for contribution costs: $N=2, c_{i} \sim_{i i d} U[-1,1]$, and $u=1$. For $q=2$, there is a unique symmetric equilibrium in which both citizens contribute and the likelihood of success is 1 . When the success threshold falls from $q=2$ to $q=1$ and citizens do not adjust their behavior, the probability of success remains 1 . So the direct effect is null. But the probability of being pivotal decreases, so each citizen contributes less, thereby reducing the likelihood of success.

One may conjecture that the logic of unanimity is the key driver of the result. If it were so, moving from $q=N-1$ to unanimity would always raise the likelihood of success for sufficiently high public good values. This conjecture is wrong. The simplicity of the example stems from the bounded support of contribution costs, not unanimity. Focusing on distributions with power-law right tails $\left(\operatorname{Pr}\left(c_{i}>x\right)=x^{-\alpha}\right.$, with $\alpha>0$, e.g., Pareto distribution), we prove that when public good value is sufficiently large, the likelihood of success increases in $q$ if and only if $\alpha>1$ (Proposition 1). When $\alpha \leq 1$, the likelihood of success is decreasing in $q$, no matter how high the public good value is even when we raise $q$ to unanimity. As we will show, the key, instead, is the rate at which $1-F$ falls.

Our results generate counterintuitive predictions in many settings. When legislators are voting to grant themselves a pay raise $(u)$, higher supermajority requirements (higher $q$ ) may lead to higher probability of a raise, especially when the raise is large relative to the public backlash from voting yes on this unpopular issue $(c) .^{2}$ Strengthening a moderately unpopular regime (raising $q$ when $u$ is moderate) makes it more likely to survive, while strengthening a more unpopular regime (raising $q$ when $u$ is large) leads to its collapse.

Our results also speak to the effect of government grants on the likelihood of threshold public good provision. Government grants in our setting correspond to reducing the success threshold $q$. Our results suggest that government grants may decrease the likelihood that a public good project succeeds. This result contrasts with the effect of government grants in the literature on public goods and charitable donations, in which the crowding out effect of a government grant never more than eliminates the value of the grant (Roberts 1984; Bergstrom, Blume, and Varian 1986; Andreoni and Payne 2003). In these models, for a fixed level of public good, a citizen's incentive to contribute weakly increases with the fraction of the good financed by the government. In contrast, in our model, the effect of government contributions on the relationship between the probability of success and citizen incentives (captured by the probability of pivotality) is more complex..$^{3}$

\footnotetext{
${ }^{2}$ Spenkuch, Montagnes, and Magleby (2018) and Shakiba (2019) provide evidence that US senators routinely behave as if it is costly for them to vote for their preferred outcome. One may worry that pivotality concerns are unlikely to drive behavior in large groups, but Spenkuch, Montagnes, and Magleby (2018) show, however, that in a chamber of 100 , US senators vote strategically based on pivotality considerations.

${ }^{3}$ These differences stem from different modeling choices. The cited models consider continuous contribution and public good levels but abstract from strategic uncertainties that arise from asymmetric information. We follow the literature with binary contribution and public good levels but allow for asymmetric information. Investigating the combination of these features is left for future work. However, two points are noteworthy. (i) The intuition for
} 
Our focus is on how variations in the success threshold $q$ affect the likelihood a public good project succeeds for a given group size $N$. In Section II, we generalize our main result, showing how the likelihood of success depends on $N-q$ and the distribution of contribution costs when the public good value $u$ is sufficiently large (Theorem 2). This result contributes to the long-standing question of the relationship between public good provision and group size, dating back to Olson (1965).

Our paper is also related to the literature on the underdog effect in elections, in which the turnout rate of the majority is lower than the minority due to higher free-riding incentives (Levine and Palfrey 2007, Krasa and Polborn 2009, Taylor and Yildirim 2010, Myatt 2015). In these models, the threshold $q$ is endogenously determined by voter turnout. They show that raising a candidate's popularity can be completely offset by free riding, but free riding does not dominate the direct effect of higher popularity, and the more popular candidate is always weakly more likely to win. ${ }^{4}$

\section{Analysis}

The strategy of citizen $i$ is a mapping from her private information $c_{i}$ to a decision whether to contribute. Fix a strategy profile of citizens other than $i, \sigma_{-i}$, and let $\operatorname{Pr}\left(p i v \mid \sigma_{-i}\right)$ be the probability that $i$ is pivotal given $\sigma_{-i}$. Then citizen $i$ with cost $c_{i}$ contributes to the project if $c_{i}<\operatorname{Pr}\left(p i v \mid \sigma_{-i}\right) u$ and does not contribute if $c_{i}>\operatorname{Pr}\left(p i v \mid \sigma_{-i}\right) u$. Thus, the best response of player $i$ is a threshold strategy. We focus on symmetric equilibria..$^{5}$ Since $F(0) \in(0,1)$, equilibrium threshold $c_{q}^{*}(u)$ is characterized by the indifference condition ${ }^{6}$

(1) $\frac{c_{q}^{*}(u)}{u}=\left(\begin{array}{c}N-1 \\ q-1\end{array}\right)\left(F\left(c_{q}^{*}(u)\right)\right)^{q-1}\left(1-F\left(c_{q}^{*}(u)\right)\right)^{N-q} \equiv \operatorname{Pr}\left(p i v \mid q, c_{q}^{*}(u)\right)$.

Let $S_{q}\left(c_{q}^{*}(u)\right)$ be the probability of success corresponding to the equilibrium threshold $c_{q}^{*}(u)$. Then,

$$
\begin{aligned}
S_{q}\left(c_{q}^{*}(u)\right) & =\sum_{k=q}^{N}\left(\begin{array}{c}
N \\
k
\end{array}\right)\left(F\left(c_{q}^{*}(u)\right)\right)^{k}\left(1-F\left(c_{q}^{*}(u)\right)\right)^{N-k} \\
& =1-\sum_{k=0}^{q-1}\left(\begin{array}{c}
N \\
k
\end{array}\right)\left(F\left(c_{q}^{*}(u)\right)\right)^{k}\left(1-F\left(c_{q}^{*}(u)\right)\right)^{N-k} .
\end{aligned}
$$

our results suggests that they qualitatively extend when the feasible contribution set is sufficiently coarse. In many applications, there are effectively few possible contribution levels, as social norms or other costs restrict contributions to a few salient levels, or the nature of contribution is discrete (e.g., volunteering time and skills for Habitat for Humanity). At the same time, asymmetric information seems to play an important role in those applications. (ii) Similar results arise in the cited models if the warm-glow payoff that citizens receive from their contributions is increasing in the contributions of other citizens but not the government's-so that government contribution can lower the marginal benefit of contributions for each level of public good.

${ }^{4}$ In Myatt (2015), a candidate who is more popular but less popular than expected will lose, but raising a candidate's realized popularity improves her chances.

${ }^{5}$ For a defense of symmetric equilibria, see Palfrey and Rosenthal (1984) and Dixit and Olson (2000).

${ }^{6}$ When the distribution of costs has bounded support, the threshold that satisfies the indifference condition may be above the upper bound of the support, in which case $F\left(c_{q}^{*}(u)\right)=1$. 
Our goal is to characterize how changes in $q$ affect the equilibrium probability of success $S_{q}\left(c_{q}^{*}(u)\right)$. For a general public good value, this analysis is intractable. We prove results for the polar opposite cases, where the public good value is sufficiently small or sufficiently large.

THEOREM 1: If the support of costs is bounded from above, or if $1-F(x)$ is log-concave for sufficiently large $x$, then the likelihood of success is increasing in the success threshold when the public good value is sufficiently large: $S_{q+1}\left(c_{q+1}^{*}(u)\right)>S_{q}\left(c_{q}^{*}(u)\right)$ for sufficiently large $u$. In contrast, the likelihood of success is always decreasing in the success threshold when the public good value is sufficiently small: $S_{q+1}\left(c_{q+1}^{*}(u)\right)<S_{q}\left(c_{q}^{*}(u)\right)$ for sufficiently small $u$.

The result is driven by the interplay of two effects. When the threshold $q$ required for success increases to $q+1$, the direct effect decreases the probability of success: fixing citizen behavior, it is less likely that at least $q+1$ of them contribute than that at least $q$ of them contribute. However, there is an indirect effect. As $q$ increases, the probability that citizen $i$ is pivotal also changes, which affects her behavior.

When the value of public good $u$ is large, each citizen contributes with large probability, so it is more likely that exactly $q$ citizens other than $i$ contribute than that exactly $q-1$ citizens other than $i$ contribute. Thus, as $q$ increases to $q+1$, citizen $i$ becomes more likely to be pivotal, and hence she increases her contribution threshold. So the indirect effect counters the direct effect. For the indirect effect to dominate, it must be that as the equilibrium threshold increases, the expected number of new participants (those whose participation $\operatorname{costs} c_{i}$ are just above the old equilibrium threshold $c_{q}^{*}$ ) is sufficiently large. When $F(c)$ increases more quickly, it means there are more such citizens; and a bounded support or log-concavity of $1-F(c)$ means that $F(c)$ increases sufficiently fast.

In contrast, when the value of public good $u$ is sufficiently small, even an increase in the probability of pivotality has a negligible effect on citizens' incentives to contribute. Therefore, the direct effect always dominates the indirect effect, so the likelihood of success decreases. 7

Theorem 1 does not address whether our result for large public good values extends to distributions with thicker right tails for which $1-F(x)$ is not log-concave for large $x$. Proposition 1 provides the analysis for the class of distributions with power-law right tails (e.g., Pareto distribution).

PROPOSITION 1: Suppose $1-F(x)=\beta / x^{\alpha}, \alpha, \beta>0$, for sufficiently large $x$. When the public good value is sufficiently large, the likelihood of success is increasing in the success threshold, $S_{q+1}\left(c_{q+1}^{*}(u)\right)>S_{q}\left(c_{q}^{*}(u)\right)$, if and only if $\alpha>1$.

Three points are worth highlighting. First, combining Theorem 1 and Proposition 1 suggests that $1 / x$ is close to the boundary tail distribution that

\footnotetext{
${ }^{7}$ When $u$ is small, each citizen contributes with probability close to $F(0)$. When $F(0)$ is small, the probability of being pivotal decreases with $q$ : if each citizen contributes with low probability, it is less likely that exactly $q$ citizens other than $i$ contribute than that exactly $q-1$ citizens other than $i$ contribute. In this case, the indirect effect strengthens the direct effect.
} 
separates cost distributions for which the strategic effect dominates (thinner than Cauchy) from those for which the direct effect dominates (thicker than Cauchy) recall that for the standard Cauchy distribution, $\operatorname{Pr}\left(c_{i}>x\right) \approx 1 / \pi x$ for large $x$.

Second, based on our simple example in the introduction, one may have thought that unanimity is the key for a higher success threshold to increase the chances of success when $u$ is sufficiently large. However, unanimity is neither necessary nor sufficient for this result. It is not sufficient because raising the success threshold from $q=N-1$ to $q+1=N$ reduces the likelihood of success when $1-F(x)=x^{-\alpha}$ for $\alpha \leq 1$, no matter how large $u$ is. It is not necessary because raising the success threshold at any level (e.g., from $q=N-3$ to $q+1=N-2$ ) increases the likelihood of success when $1-F(x)$ goes to 0 faster and $u$ is sufficiently large. ${ }^{8}$

Third, a higher success threshold increases the chances of success only if it raises the contribution threshold: $S_{q+1}\left(c_{q+1}^{*}\right)>S_{q}\left(c_{q}^{*}\right)$ requires $c_{q+1}^{*}>c_{q}^{*}$. This may lead one to conjecture that a very large $c_{q+1}^{*} / c_{q}^{*}$ is sufficient for $S_{q+1}\left(c_{q+1}^{*}\right)>S_{q}\left(c_{q}^{*}\right)$. This conjecture is incorrect. To see this, suppose $1-F(x)=x^{-\alpha}$ with $\alpha>0$ for large $x$, and compare $c_{N}^{*}(u)$ and $c_{1}^{*}(u)$ when $u$ is very large. The indifference conditions imply

$$
\lim _{u \rightarrow \infty} \frac{c_{N}^{*}(u)}{u}=1 \quad \text { and } \quad \lim _{u \rightarrow \infty} \frac{c_{1}^{*}(u)}{u^{\frac{1}{1+\alpha(N-1)}}}=1 .
$$

Thus, $c_{N}^{*}(u) / c_{1}^{*}(u)$ can be made arbitrary large by choosing a sufficiently large $u$. However, Proposition 1 shows that no matter how large $u$ becomes, $S_{N}\left(c_{N}^{*}(u)\right)<S_{1}\left(c_{1}^{*}(u)\right)$ for $\alpha \in(0,1]$. In fact, when $q \in\{1, \ldots, N-2\}$, for $S_{q+1}\left(c_{q+1}^{*}(u)\right)>S_{q}\left(c_{q}^{*}(u)\right)$ to hold for sufficiently large $u, c_{q+1}^{*} / c_{q}^{*}$ must not be too large. $\rho^{9}$ To see this, consider equations (1) and (3) and suppose $u$ is very large. A citizen almost always contributes, so that the dominant term in these equations is $1-F\left(c_{q}^{*}(u)\right)$ with the lowest power

$$
\operatorname{Pr}\left(p i v \mid q, c_{q}^{*}\right) \approx\left(\begin{array}{c}
N-1 \\
q-1
\end{array}\right)\left(1-F\left(c_{q}^{*}\right)\right)^{N-q}
$$

and

$$
S_{q}\left(c_{q}^{*}\right) \approx 1-\left(\begin{array}{c}
N \\
q-1
\end{array}\right)\left(1-F\left(c_{q}^{*}\right)\right)^{N-q+1}
$$

For $S_{q+1}\left(c_{q+1}^{*}\right)>S_{q}\left(c_{q}^{*}\right)$, we want $\left(1-F\left(c_{q+1}^{*}\right)\right)^{N-q}$ to be as small as possible. But this does not mean that we want $c_{q+1}^{*}$ to be as large as possible, because $c_{q+1}^{*}$ is an equilibrium object and is linked to $\left(1-F\left(c_{q+1}^{*}\right)\right)^{N-q}$ through the likelihood of pivotality: in equilibrium, if $c_{q+1}^{*}$ is higher, then so is $1-F\left(c_{q+1}^{*}\right)$. Rather, as Theorem 1 and Proposition 1 show, the key is how fast $1-F$ falls. When

\footnotetext{
${ }^{8}$ Moreover, note that for all $q \in\{1, \ldots, N-1\}$, unlike for unanimity, each player is unlikely to be pivotal even when $u$ is very large, while each player contributes with probability close to 1 .

${ }^{9}$ See part (iii) of Lemma 1 in the Appendix.
} 
Panel A

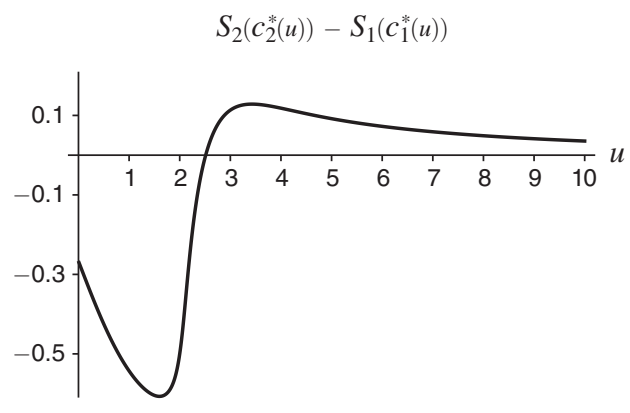

Panel B

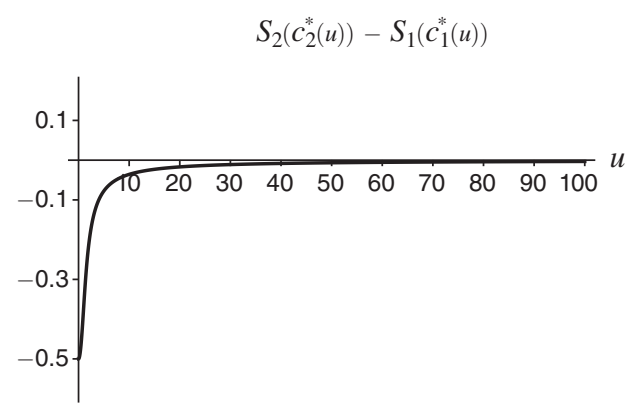

FIGURE 2

Note: The difference between success probability when $q=2$ and when $q=1$ for a game with two players when the distribution of costs is $N(1,1)$ (panel A) and Cauchy with location 0 and scale 1 (panel $\mathrm{B}$ ).

raising $q$ to $q+1$, if the equilibrium contribution threshold did not change, we would have

$$
\left(\begin{array}{c}
N-1 \\
q
\end{array}\right)\left(1-F\left(c_{q}^{*}\right)\right)^{N-(q+1)}>\left(\begin{array}{c}
N-1 \\
q-1
\end{array}\right)\left(1-F\left(c_{q}^{*}\right)\right)^{N-q} \approx \frac{c_{q}^{*}}{u} .
$$

But now the equilibrium contribution threshold increases to $c_{q+1}^{*}$ to restore indifference:

$$
\left(\begin{array}{c}
N-1 \\
q
\end{array}\right)\left(1-F\left(c_{q+1}^{*}\right)\right)^{N-(q+1)} \approx \frac{c_{q+1}^{*}}{u}
$$

It follows that the faster $1-F(\cdot)$ drops around $c_{q}^{*}$, the lower will be $1-F\left(c_{q+1}^{*}\right)$, and hence the higher will be $S_{q+1}\left(c_{q+1}^{*}\right)$.

Our analytical results focus on the asymptotic cases when the public good value is very small or very large. How does the likelihood of success vary with the success threshold for public good values away from the extremes? To glean insights, we provide simulations. First, consider the example in the introduction but with normal and Cauchy distributions. Figure 2 illustrates. With $F=N(1,1)$, raising the success threshold from 1 to 2 increases the likelihood of success whenever the public good value $u$ is above a threshold of about 2.52. Around this public good value, for $q=2$, a citizen participates and is pivotal with probability 0.90 . In contrast, with $F=\operatorname{Cauch} y(0,1)$, raising the success threshold always reduces the likelihood of success, for example, even when $u=100$ at which a citizen participates with probability 0.9968 and is pivotal with probability 0.997 under unanimityfor $q=1$, each citizen is pivotal with probability 0.056 . Next, suppose $N=3$, and raise the success threshold from $q=1$ to $q=2$. For $F=N(2,1)$, when the public good value is higher than 8.11, again, the likelihood of success falls. Around this public good value, for $q=2$, a citizen participates with probability 0.78 and is pivotal with probability 0.34 . For $F=\operatorname{Cauchy}(0,1)$, at $u=8.1$, the probability of 
pivotality is 0.25 when $q=2$ and 0.92 when $q=3$, and the probability of success always decreases in $q$. $^{10}$

These simulations further confirm that unanimity, a high probability of pivotality, or a very high probability of participation are not the driving force behind our results. They do indicate, however, that the public good value has to be large relative to individual contribution costs. Indeed, in many applications, the public good value is far larger than a typical individual's contribution costs. For example, using the data from Texas local liquor referendums, Coate, Conlin, and Moro (2008) estimate the average value of passage to potential voters as 35 times larger than the average voting costs. In the applications mentioned in the introduction (e.g., reporting a crime, Palfrey and Rosenthal 1984, or boycotting a product, Diermeier and Van Mieghem 2008), one expects that the public good value is large enough relative to individual participation costs so that any player would be willing to pay her participation costs if she were the only player and her participation would deliver the good.

\section{Generalized Theorem}

Our intuition suggests a close link between the effect of raising the success threshold $q$ and reducing the group size $N$. We now extend Theorem 1, showing how the likelihood of success depends on $N-q$. We state the generalized theorem below and provide its proof and the analogous generalization of Proposition 1 in an online Appendix. Let $c_{q, N}^{*}(u)$ be the symmetric equilibrium threshold in the game with success threshold $q$ and $N$ players.

THEOREM 2: Take $q, N, q^{\prime}$, and $N^{\prime}$ such that $q \leq N$ and $q^{\prime} \leq N^{\prime}$.

(i) If $q \leq q^{\prime}$ and $N-q \geq N^{\prime}-q^{\prime}$, with at least one inequality strict, then $S_{q, N}\left(c_{q, N}^{*}(u)\right)>S_{q^{\prime}, N^{\prime}}\left(c_{q^{\prime}, N^{\prime}}^{*}(u)\right)$ for sufficiently small $u$.

(ii) Suppose the support of costs is bounded from above, or $1-F(x)$ is log-concave for sufficiently large $x$. If $N-q<N^{\prime}-q^{\prime}$, then $S_{q, N}\left(c_{q, N}^{*}(u)\right)>S_{q^{\prime}, N^{\prime}}\left(c_{q^{\prime}, N^{\prime}}^{*}(u)\right)$ for sufficiently large $u$.

Theorem 2 sheds light on the long-standing question of the relationship between group size and public good provision. Olson (1965) argued that larger groups are less likely to provide public goods because they have a more severe free-riding problem. However, Chamberlin (1974) (and many since) showed that Olson's conjecture does not hold in standard public good models like Bergstrom, Blume, and Varian (1986). ${ }^{11}$ The direct effect of raising group size dominates the strategic free-riding effect so that larger groups provide more public goods. In symmetric mixed strategy equilibria of the complete-information threshold public good game with $q=1$ (analyzed in Palfrey and Rosenthal 1984), Diekmann (1985) showed that the

\footnotetext{
${ }^{10}$ There is a unique symmetric equilibrium for the parameters of our simulations.

${ }^{11}$ In Esteban and Ray (2001), multiple groups compete for a prize. When the prize is a purely public good, the probability of success increases in group size.
} 
probability of success is decreasing in group size $N$. We show this result is not robust to the strategic uncertainty introduced by private participation costs. ${ }^{12}$ When the public good value is small, the likelihood of public good provision is increasing in $N$. In contrast, for sufficiently valuable public goods, the strategic effect dominates, and Olson's conjecture holds for any success threshold $q \leq N$ if either the distribution of contribution costs are bounded from above or have log-concave right tails. Our generalization of Proposition 1 (in the online Appendix) shows that when the right-tail distribution of participation costs is fatter than Cauchy, Olson's conjecture is reversed even for very high-value public goods. 13

\section{Conclusion}

Public good projects that require fewer contributors to succeed may seem to have a higher chance of success. That fewer contributors are needed for success may increase free-riding incentives, but common sense suggests that this strategic effect should not offset the direct effect. We show that in a standard threshold public good game with binary contributions, this intuition fails under quite standard conditions.

Two directions for future research stand out. (i) We analyzed the case in which costs are uncorrelated. Shadmehr (forthcoming) provides a partial characterization of the same game in the limit when the number of citizens is large and costs have common and idiosyncratic components. With a numerical example, he shows that with normal prior about the common cost, the likelihood of success can be increasing in the success threshold. Analyzing the problem for intermediate correlation levels remains to be done. (ii) The simple setting makes testing predictions in lab experiments feasible, and valuable given the counterintuitive nature of the results.

\section{APPENDIX: ProOfS}

To ease the exposition, we drop superscript in $c_{q}^{*}(u)$ and write $c_{q}(u)$ or $c_{q}$ for equilibrium thresholds, recognizing that $c_{q}$ depends on model parameters, including $u$. We also use $S_{q}(u)$ for $S_{q}\left(c_{q}^{*}(u)\right)$.

We begin with a preliminary lemma that will be used in the proof of Theorem 1. Part (iii) of this lemma bounds $c_{q+1} / c_{q}$ as $u \rightarrow \infty$. As discussed in the main text, ensuring that $c_{q+1} / c_{q}$ is not too large is crucial for the argument.

LEMMA 1: $(i) \lim _{u \rightarrow \infty} c_{N}(u) / u=1$. Moreover, if the support of costs is bounded from above, or if $1-F(x)$ is log-concave for sufficiently large $x$, then (ii) for

\footnotetext{
${ }^{12}$ In particular, consider $c_{i}=c+\sigma \epsilon_{i}$. For any $\sigma$, Diekmann's result is overturned for sufficiently small $u$ if $F\left(c_{i}\right)$ satisfies our conditions and for sufficiently large $u$ if the distribution of $\epsilon_{i}$ has fat tails. In a dynamic extension of Palfrey and Rosenthal (1984, 1988), Yildirim (2006) finds that in later periods when dynamic incentives are absent, participation is decreasing in group size when participation costs have bounded support.

${ }^{13}$ Deserranno, Nansamba, and Qian (2020) look at a random entry of nongovernmental organizations into areas of Uganda that already have government-provided health-care workers. The entry of nongovernmental organizations reduces the probability of receiving health care from any health-care worker (as measured by whether a household "received medical care" in the past year) by 12 percentage points. This finding is consistent with part (ii) of Theorem 2 in that increasing the number of potential health-care providers leads to a lower probability of health-care provision.
} 
all $\alpha>0, \lim _{u \rightarrow \infty} c_{q}(u) / u^{\alpha}=0$ if $q<N$, and $(i i i) \lim _{u \rightarrow \infty} c_{q+1}(u) / c_{q}(u)<\infty$ if $q<N-1$.

\section{PROOF:}

Part (i) directly follows from (1).

To prove parts (ii) and (iii), suppose first the support of costs is bounded from above. Then for $q<N, c_{q}(u)$ is strictly smaller than the upper bound of the cost distribution, so it is bounded from above (which proves part (ii)) and bounded away from 0 (which proves part (iii)).

Next, suppose the support of costs does not have an upper bound, but $1-F(x)$ is log-concave for sufficiently large $x$.

Part (ii)._-Differentiating (1) with respect to $u$ yields

$$
\begin{aligned}
& \text { (4) } \frac{d c_{q}(u)}{d u} \\
& =\frac{\left(\begin{array}{c}
N-1 \\
q-1
\end{array}\right)\left(F\left(c_{q}\right)\right)^{q-1}\left(1-F\left(c_{q}\right)\right)^{N-q}}{1-u\left(\begin{array}{c}
N-1 \\
q-1
\end{array}\right)\left(F\left(c_{q}\right)\right)^{q-2}\left(1-F\left(c_{q}\right)\right)^{N-q-1}\left((q-1)\left(1-F\left(c_{q}\right)\right)-(N-q) F\left(c_{q}\right)\right) f\left(c_{q}\right)} .
\end{aligned}
$$

From (1), the numerator is equal to $c_{q} / u$, and also

$$
\left(\begin{array}{c}
N-1 \\
q-1
\end{array}\right)\left(F\left(c_{q}\right)\right)^{q-2}\left(1-F\left(c_{q}\right)\right)^{N-q-1}=\frac{c_{q}(u)}{u} \frac{1}{F\left(c_{q}\right)\left(1-F\left(c_{q}\right)\right)} .
$$

Substituting $c_{q} / u$ and (5) into (4) yields

$$
\text { (6) } \begin{aligned}
\frac{d c_{q}(u)}{d u} & =\frac{\frac{c_{q}}{u}}{1-u \frac{c_{q}}{u} \frac{1}{F\left(c_{q}\right)\left(1-F\left(c_{q}\right)\right)}\left((q-1)\left(1-F\left(c_{q}\right)\right)-(N-q) F\left(c_{q}\right)\right) f\left(c_{q}\right)} \\
& =\frac{1}{u} \frac{1}{\frac{1}{c_{q}}-\left((q-1) \frac{1-F\left(c_{q}\right)}{F\left(c_{q}\right)}-(N-q)\right) \frac{f\left(c_{q}\right)}{1-F\left(c_{q}\right)}} .
\end{aligned}
$$

From $(1), \lim _{u \rightarrow \infty} c_{q}(u)=\infty$, and hence $\lim _{u \rightarrow \infty} F\left(c_{q}(u)\right)=1$. Using L'Hôpital's rule, we have

$$
\begin{aligned}
\lim _{u \rightarrow \infty} \frac{c_{q}(u)}{u^{\alpha}} & =\lim _{u \rightarrow \infty} \frac{\frac{d c_{q}(u)}{d u}}{\alpha u^{\alpha-1}} \\
& =\lim _{u \rightarrow \infty} \frac{1}{u} \frac{1}{\frac{1}{c_{q}}-\left((q-1) \frac{1-F\left(c_{q}\right)}{F\left(c_{q}\right)}-(N-q)\right) \frac{f\left(c_{q}\right)}{1-F\left(c_{q}\right)}} \frac{1}{\alpha u^{\alpha-1}} \quad(\text { from }(6)) \\
& =\lim _{u \rightarrow \infty} \frac{1}{\alpha} \frac{1}{1-\left((q-1) \frac{1-F\left(c_{q}\right)}{F\left(c_{q}\right)}-(N-q)\right) \frac{f\left(c_{q}\right)}{1-F\left(c_{q}\right)} c_{q}} \frac{c_{q}}{u^{\alpha}} .
\end{aligned}
$$


Since $1-F$ is log-concave, $\lim _{u \rightarrow \infty} f\left(c_{q}(u)\right) /\left(1-F\left(c_{q}(u)\right)\right)>0$, and the second line above implies that $\lim _{u \rightarrow \infty} c_{q}(u) / u^{\alpha}<\infty$, so we can move $\lim _{u \rightarrow \infty} c_{q}(u) / u^{\alpha}$ to one side, obtaining

$$
\lim _{u \rightarrow \infty} \frac{c_{q}(u)}{u^{\alpha}}\left(1-\frac{1}{\alpha} \frac{1}{1-\left((q-1) \frac{1-F\left(c_{q}\right)}{F\left(c_{q}\right)}-(N-q)\right) \frac{f\left(c_{q}\right)}{1-F\left(c_{q}\right)} c_{q}}\right)=0
$$

Because $\lim _{u \rightarrow \infty} f\left(c_{q}\right) /\left(1-F\left(c_{q}\right)\right)>0$, the limit of the term in parentheses is not 0 for any $\alpha$, and hence $\lim _{u \rightarrow \infty} c_{q}(u) / u^{\alpha}=0$.

Part (iii).-By contradiction, suppose that $\lim _{u \rightarrow \infty} c_{q+1}(u) / c_{q}(u)=\infty$. Then, $c_{q+1}(u)>c_{q}(u)$ for sufficiently large $u$. Because $1-F$ is log-concave, $f /(1-F)$ is increasing, and hence

$$
\lim _{u \rightarrow \infty} \frac{\frac{f\left(c_{q}(u)\right)}{1-F\left(c_{q}(u)\right)}}{\frac{f\left(c_{q+1}(u)\right)}{1-F\left(c_{q+1}(u)\right)}}<\infty
$$

Moreover, using L'Hôpital's rule, we obtain

$$
\begin{aligned}
\lim _{u \rightarrow \infty} \frac{c_{q+1}(u)}{c_{q}(u)} & =\lim _{u \rightarrow \infty} \frac{\frac{d c_{q+1}(u)}{d u}}{\frac{d c_{q}(u)}{d u}} \\
& \left.=\lim _{u \rightarrow \infty} \frac{\frac{1}{c_{q}}-\left((q-1) \frac{1-F\left(c_{q}\right)}{F\left(c_{q}\right)}-(N-q)\right) \frac{f\left(c_{q}\right)}{1-F\left(c_{q}\right)}}{\frac{1}{c_{q+1}}-\left(q \frac{1-F\left(c_{q+1}\right)}{F\left(c_{q+1}\right)}-(N-q-1)\right) \frac{f\left(c_{q+1}\right)}{1-F\left(c_{q+1}\right)}} \quad \text { (from }(6)\right) \\
& \left.=\lim _{u \rightarrow \infty} \frac{N-q}{N-q-1} \frac{\frac{f\left(c_{q}(u)\right)}{1-F\left(c_{q}(u)\right)}}{\frac{f\left(c_{q+1}(u)\right)}{1-F\left(c_{q+1}(u)\right)}}<\quad \text { (from }(7)\right),
\end{aligned}
$$

which is a contradiction.

We now provide the proof of Theorem 1.

\section{PROOF OF THEOREM 1:}

First, we prove the second part of the theorem pertaining to low $u$. From (1), $c_{q}(0)=c_{q+1}(0)=0$. Thus, from (2),

$$
\begin{aligned}
S_{q+1}(0)-S_{q}(0)= & \sum_{k=q+1}^{N}\left(\begin{array}{l}
N \\
k
\end{array}\right)(F(0))^{k}(1-F(0))^{N-k} \\
& -\sum_{k=q}^{N}\left(\begin{array}{l}
N \\
k
\end{array}\right)(F(0))^{k}(1-F(0))^{N-k}
\end{aligned}
$$




$$
=-\left(\begin{array}{c}
N \\
q
\end{array}\right)(F(0))^{q}(1-F(0))^{N-q}<0
$$

The result follows from the continuity of $c_{q}(u)$ and $S_{q}(u)$ in $u$.

Now, we prove the first part of the theorem. The difference between success probability for $q+1$ and $q$ is as follows:

$$
S_{q+1}(u)-S_{q}(u)=\left(1-S_{q}(u)\right)\left(1-\frac{1-S_{q+1}(u)}{1-S_{q}(u)}\right) .
$$

Thus, it suffices to show

$$
\lim _{u \rightarrow \infty} \frac{1-S_{q+1}(u)}{1-S_{q}(u)}<1 .
$$

From (3),

$$
\begin{aligned}
\frac{1-S_{q+1}(u)}{1-S_{q}(u)} & =\frac{\sum_{k=0}^{q}\left(\begin{array}{l}
N \\
k
\end{array}\right)\left(F\left(c_{q+1}\right)\right)^{k}\left(1-F\left(c_{q+1}\right)\right)^{N-k}}{\sum_{k=0}^{q-1}\left(\begin{array}{l}
N \\
k
\end{array}\right)\left(F\left(c_{q}\right)\right)^{k}\left(1-F\left(c_{q}\right)\right)^{N-k}} \\
& =\frac{\sum_{k=0}^{q}\left(\begin{array}{l}
N \\
k
\end{array}\right)\left(F\left(c_{q+1}\right)\right)^{k}\left(1-F\left(c_{q+1}\right)\right)^{q-k}\left(1-F\left(c_{q+1}\right)\right)^{N-q}}{\sum_{k=0}^{q-1}\left(\begin{array}{l}
N \\
k
\end{array}\right)\left(F\left(c_{q}\right)\right)^{k}\left(1-F\left(c_{q}\right)\right)^{q-1-k}\left(1-F\left(c_{q}\right)\right)^{N-q+1}} .
\end{aligned}
$$

Moreover, since $\lim _{u \rightarrow \infty} F\left(c_{q}\right)=\lim _{u \rightarrow \infty} F\left(c_{q+1}\right)=1$,

$$
\begin{aligned}
& \lim _{u \rightarrow \infty} \sum_{k=0}^{q}\left(\begin{array}{c}
N \\
k
\end{array}\right)\left(F\left(c_{q+1}\right)\right)^{k}\left(1-F\left(c_{q+1}\right)\right)^{q-k}=\left(\begin{array}{c}
N \\
q
\end{array}\right), \\
& \lim _{u \rightarrow \infty} \sum_{k=0}^{q-1}\left(\begin{array}{c}
N \\
k
\end{array}\right)\left(F\left(c_{q}\right)\right)^{k}\left(1-F\left(c_{q}\right)\right)^{q-1-k}=\left(\begin{array}{c}
N \\
q-1
\end{array}\right) .
\end{aligned}
$$

Substituting (9) and (10) into (8) yields

$$
\lim _{u \rightarrow \infty} \frac{1-S_{q+1}(u)}{1-S_{q}(u)}=\lim _{u \rightarrow \infty} \frac{\left(\begin{array}{c}
N \\
q
\end{array}\right)}{\left(\begin{array}{c}
N \\
q-1
\end{array}\right)} \frac{\left(1-F\left(c_{q+1}\right)\right)^{N-q}}{\left(1-F\left(c_{q}\right)\right)^{N-q+1}} .
$$

For the rest of the proof, we consider two cases separately: $q<N-1$ and $q=N-1$. The latter case corresponds to moving to unanimity, and part (iii) of Lemma 1 does not apply for this case. Therefore, it requires a different approach.

Case $q<N-1$ : From the indifference condition (1),

$$
\left(1-F\left(c_{q}\right)\right)^{N-q+1}=\left(\frac{c_{q}}{u} \frac{1}{\left(\begin{array}{c}
N-1 \\
q-1
\end{array}\right)\left(F\left(c_{q}\right)\right)^{q-1}}\right)^{\frac{N-q+1}{N-q}}, \text { for } q \in\{1, \ldots, N-1\}
$$




$$
\left(1-F\left(c_{q+1}\right)\right)^{N-q}=\left(\frac{c_{q+1}}{u} \frac{1}{\left(\begin{array}{c}
N-1 \\
q
\end{array}\right)\left(F\left(c_{q+1}\right)\right)^{q}}\right)^{\frac{N-q}{N-q-1}}, \text { for } q \in\{1, \ldots, N-2\} .
$$

Substituting these into (11) yields that, for $q \in\{1, \ldots, N-2\}$,

$$
\begin{aligned}
& \lim _{u \rightarrow \infty} \frac{1-S_{q+1}(u)}{1-S_{q}(u)}=\lim _{u \rightarrow \infty} \frac{N-q+1}{q} \frac{\left(\frac{c_{q+1}(u)}{u} \frac{1}{\left(\begin{array}{c}
N-1 \\
q
\end{array}\right)\left(F\left(c_{q+1}(u)\right)\right)^{q}}\right)^{\frac{N-q}{N-q-1}}}{\left(\frac{c_{q}(u)}{u} \frac{1}{\left(\begin{array}{c}
N-1 \\
q-1
\end{array}\right)\left(F\left(c_{q}(u)\right)\right)^{q-1}}\right)^{\frac{N-q+1}{N-q}}} \\
& =\lim _{u \rightarrow \infty} \frac{N-q+1}{q} \frac{\left(\frac{1}{\left(\begin{array}{c}
N-1 \\
q
\end{array}\right)\left(F\left(c_{q+1}(u)\right)\right)^{q}}\right)^{\frac{N-q}{N-q-1}}}{\left(\frac{1}{\left(\begin{array}{c}
N-1 \\
q-1
\end{array}\right)\left(F\left(c_{q}(u)\right)\right)^{q-1}}\right)^{\frac{N-q+1}{N-q}}}\left(\frac{c_{q+1}(u)}{c_{q}(u)}\right)^{\frac{N-q+1}{N-q}} \\
& \times\left(\frac{c_{q+1}(u)}{u}\right)^{\frac{1}{(N-q-1)(N-q)}} \\
& =\frac{N-q+1\left(\frac{1}{\left(\begin{array}{c}
N-1 \\
q
\end{array}\right)}\right)^{\frac{N-q}{N-q-1}}}{\left(\frac{1}{\left(\begin{array}{c}
N-1 \\
q-1
\end{array}\right)}\right)^{\frac{N-q+1}{N-q}}\left(\lim _{u \rightarrow \infty} \frac{c_{q+1}(u)}{c_{q}(u)}\right)^{\frac{N-q+1}{N-q}}} \\
& \times\left(\lim _{u \rightarrow \infty} \frac{c_{q+1}(u)}{u}\right)^{\frac{1}{(N-q-1)(N-q)}} .
\end{aligned}
$$

From part (ii) of Lemma $1, \lim _{u \rightarrow \infty} c_{q+1}(u) / u=0$. From part (iii) of Lemma $1, \lim _{u \rightarrow \infty} c_{q+1}(u) / c_{q}(u)<\infty$. Thus, $\lim _{u \rightarrow \infty}\left(1-S_{q+1}(u)\right) /\left(1-S_{q}(u)\right)$ $=0$, and the result follows.

Case $q=N-1:$ From (11), it suffices to show

$$
\lim _{u \rightarrow \infty} \frac{1-S_{N}(u)}{1-S_{N-1}(u)}=\lim _{u \rightarrow \infty} \frac{2}{N-1} \frac{1-F\left(c_{N}\right)}{\left(1-F\left(c_{N-1}\right)\right)^{2}}<1
$$

We show $\lim _{u \rightarrow \infty}\left(1-F\left(c_{N}\right)\right) /\left(1-F\left(c_{N-1}\right)\right)^{2}=0$. If the support of costs is bounded from above, then $1-F\left(c_{N}(u)\right)=0$ for sufficiently large $u$, while $1-$ $F\left(c_{N-1}(u)\right)>0$ for all $u$, although it becomes arbitrarily close to 0 . Next, consider 
the case where the support of costs has no upper bound. From parts (i) and (ii) of Lemma 1, it suffices to show

$$
\lim _{u \rightarrow \infty} \frac{1-F(u)}{\left(1-F\left(u^{\alpha}\right)\right)^{2}}=0, \quad \text { for some } \alpha \in(0,1) .
$$

Because $1-F(x) \in(0,1)$ is decreasing and log-concave, its right tail is at most exponential (An 1998). In particular, for sufficiently large $x, 1-F(x)$ goes to 0 faster than $x^{-b}$, for any $b>0$.

Pick an $\alpha \in(0,1 / 2)$. Fix $\epsilon>0$. Pick a sufficiently large $\hat{x}$, such that for $x>\hat{x}, 1-F(x)$ goes to 0 faster than $x^{-b}$, for any $b>0$. Pick $x_{1}>\hat{x}$, and find $b_{1}$ such that $1-F\left(x_{1}^{\alpha}\right)=\left(x_{1}^{\alpha}\right)^{-b_{1}}=x_{1}^{-\alpha b_{1}}$. Hence, $1-F\left(x_{1}\right)<x_{1}^{-b_{1}}$, and hence

$$
\frac{1-F\left(x_{1}\right)}{\left(1-F\left(x_{1}^{\alpha}\right)\right)^{2}}=\frac{1-F\left(x_{1}\right)}{x_{1}^{-2 \alpha b_{1}}}<\frac{x_{1}^{-b_{1}}}{x_{1}^{-2 \alpha b_{1}}}=\frac{1}{x_{1}^{b_{1}(1-2 \alpha)}} .
$$

If $1 / x_{1}^{b_{1}(1-2 \alpha)}<\epsilon$, then set $\bar{x}=\epsilon^{-\frac{1}{(1-2 \alpha) b_{1}}}$. Otherwise, choose $\bar{x}>x_{1}$ large enough, so that $1 / \bar{x}^{b_{1}(1-2 \alpha)}<\epsilon$. Because $1-F(x)$ goes to 0 faster than $x^{-b_{1}}$, there exists a $b_{2}>b_{1}$ such that $1-F\left(\bar{x}^{\alpha}\right)=\left(\bar{x}^{\alpha}\right)^{-b_{2}}=\bar{x}^{-\alpha b_{2}}$. Thus,

$$
\frac{1-F(\bar{x})}{\left(1-F\left(\bar{x}^{\alpha}\right)\right)^{2}}=\frac{1-F(\bar{x})}{\bar{x}^{-2 \alpha b_{2}}}<\frac{\bar{x}^{-b_{2}}}{\bar{x}^{-2 \alpha b_{2}}}=\frac{1}{\bar{x}^{b_{2}(1-2 \alpha)}}<\frac{1}{\bar{x}^{b_{1}(1-2 \alpha)}}<\epsilon .
$$

Now, consider $x>\bar{x}$. Find $b_{3}$, so that $1-F\left(x^{\alpha}\right)=x^{-\alpha b_{3}}$. Again, because $1-F(x)$ goes to 0 faster than $x^{-b}$, for all $b>0, b_{3}>b_{2}$, and hence $(1-F(x)) /\left(1-F\left(x^{\alpha}\right)\right)^{2}<\epsilon$. The result follows.

We have shown that for any given $q \in\{1, \ldots, N-1\}, S_{q+1}(u)>S_{q}(u)$ for sufficiently large $u$. Because there is a finite number of $q \mathrm{~s}, S_{q}(u)$ increases in $q$ for sufficiently large $u$.

\section{PROOF OF PROPOSITION 1:}

Substituting $1-F(x)=\beta / x^{\alpha}$ in (11) yields

$$
\lim _{u \rightarrow \infty} \frac{1-S_{q+1}(u)}{1-S_{q}(u)}=\lim _{u \rightarrow \infty} \frac{N-q+1}{\beta q}\left(\frac{\left(c_{q}(u)\right)^{N-q+1}}{\left(c_{q+1}(u)\right)^{N-q}}\right)^{\alpha} .
$$

From indifference condition (1),

$$
c_{q}(u)=\left(\begin{array}{c}
N-1 \\
q-1
\end{array}\right)\left(1-\frac{\beta}{\left(c_{q}(u)\right)^{\alpha}}\right)^{q-1}\left(\frac{\beta}{\left(c_{q}(u)\right)^{\alpha}}\right)^{N-q} u .
$$

Thus,

$$
\left(c_{q}(u)\right)^{N-q+1}=\left(\left(\begin{array}{c}
N-1 \\
q-1
\end{array}\right) \beta^{N-q}\left(1-\frac{\beta}{\left(c_{q}(u)\right)^{\alpha}}\right)^{q-1} u\right)^{\frac{N-q+1}{\alpha(N-q)+1}} .
$$


Substituting this in (14) yields

$$
\begin{aligned}
& \lim _{u \rightarrow \infty} \frac{1-S_{q+1}(u)}{1-S_{q}(u)} \\
& =\lim _{u \rightarrow \infty} \frac{N-q+1}{\beta q}\left(\frac{\left(\left(\begin{array}{c}
N-1 \\
q-1
\end{array}\right) \beta^{N-q}\left(1-\frac{\beta}{\left(c_{q}(u)\right)^{\alpha}}\right)^{q-1} u\right)^{\frac{N-q+1}{\alpha(N-q)+1}}}{\left(\left(\begin{array}{c}
N-1 \\
q
\end{array}\right) \beta^{N-q-1}\left(1-\frac{\beta}{\left(c_{q+1}(u)\right)^{\alpha}}\right)^{q} u\right)^{\frac{N-q}{\alpha(N-q-1)+1}}}\right)^{\alpha} \\
& =\lim _{u \rightarrow \infty} \frac{N-q+1}{\beta q}\left(\frac{\left(\left(\begin{array}{c}
N-1 \\
q-1
\end{array}\right) \beta^{N-q} u\right)^{\frac{N-q+1}{\alpha(N-q)+1}}}{\left(\left(\begin{array}{c}
N-1 \\
q
\end{array}\right) \beta^{N-q-1} u\right)^{\frac{N-q}{\alpha(N-q-1)+1}}}\right)^{\alpha} \\
& =\lim _{u \rightarrow \infty} \frac{N-q+1}{\beta q}\left(\frac{\left(\left(\begin{array}{c}
N-1 \\
q-1
\end{array}\right) \beta^{N-q}\right)^{\frac{N-q+1}{\alpha(N-q)+1}}}{\left(\left(\begin{array}{c}
N-1 \\
q
\end{array}\right) \beta^{N-q-1}\right)^{\frac{N-q}{\alpha(N-q-1)+1}}}\right)^{\alpha} u^{\left(\frac{N-q+1}{\alpha(N-q)+1}-\frac{N-q}{\alpha(N-q-1)+1}\right) \alpha} .
\end{aligned}
$$

Write the power of $u$ as $\frac{\alpha}{(\alpha(N-q)+1)(\alpha(N-q-1)+1)}(1-\alpha)$, and recognize that $\frac{\alpha}{(\alpha(N-q)+1)(\alpha(N-q-1)+1)}>0$. Thus,

$$
\lim _{u \rightarrow \infty} \frac{1-S_{q+1}(u)}{1-S_{q}(u)}= \begin{cases}0, & \alpha>1 \\ \infty, & \alpha<1\end{cases}
$$

and hence

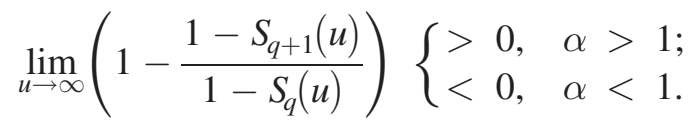

When $\alpha=1$,

$$
\lim _{u \rightarrow \infty} \frac{1-S_{q+1}(u)}{1-S_{q}(u)}=\frac{N-q+1}{\beta q} \frac{\left(\begin{array}{c}
N-1 \\
q-1
\end{array}\right) \beta^{N-q}}{\left(\begin{array}{c}
N-1 \\
q
\end{array}\right) \beta^{N-q-1}}=\frac{N-q+1}{N-q} .
$$

Thus, for $\alpha=1, \lim _{u \rightarrow \infty}\left(1-\frac{1-S_{q+1}(u)}{1-S_{q}(u)}\right)=-\frac{1}{N-q}<0$. 


\section{REFERENCES}

An, Mark Yuying. 1998. "Logconcavity versus Logconvexity: A Complete Characterization.” Journal of Economic Theory 80 (2): 350-69.

-Andreoni, James, and A. Abigail Payne. 2003. "Do Government Grants to Private Charities Crowd out Giving or Fund-Raising?" American Economic Review 93 (3): 792-812.

Bergstrom, Theodore, Larry Blume, and Hal Varian. 1986. "On the Private Provision of Public Goods." Journal of Public Economics 29 (1): 25-49.

Cadsby, Charles Bram, and Elizabeth Maynes. 1999. "Voluntary Provision of Threshold Public Goods with Continuous Contributions: Experimental Evidence." Journal of Public Economics 71 (1): 53-73.

Chamberlin, John. 1974. "Provision of Collective Goods as a Function of Group Size." American Political Science Review 68 (2): 707-16.

Coate, Stephen, Michael Conlin, and Andrea Moro. 2008. "The Performance of Pivotal-Voter Models in Small-Scale Elections: Evidence from Texas Liquor Referenda." Journal of Public Economics 92 (3-4): 582-96.

Dawes, Robyn M., John M. Orbell, Randy T. Simmons, and Alphons J. C. Van De Kragt. 1986. "Organizing Groups for Collective Action." American Political Science Review 80 (4): 1171-85.

Deserranno, Erika, Aisha Nansamba, and Nancy Qian. 2020. "Aid Crowd-out: The Effect of NGOs on Government-Provided Public Services.” National Bureau of Economic Research Working Paper 26928.

Diekmann, Andreas. 1985. "Volunteer's Dilemma." Journal of Conflict Resolution 29 (4): 605-10.

Diermeier, Daniel, and Jan A. Van Mieghem. 2008. "Voting with Your Pocketbook-A Stochastic Model of Consumer Boycotts." Mathematical and Computer Modelling 48 (9-10): 1497-1509.

Dixit, Avinash, and Mancur Olson. 2000. "Does Voluntary Participation Undermine the Coase Theorem?" Journal of Public Economics 76 (3): 309-35.

Esteban, Joan, and Debraj Ray. 2001. "Collective Action and the Group Size Paradox." American Political Science Review 95 (3): 663-72.

Fudenberg, Drew, and Jean Tirole. 1991. "Example 6.1: Providing a Public Good under Incomplete Information." In Game Theory, 211-12. Cambridge: MIT Press.

Goeree, Jacob K., and Charles A. Holt. 2005. "An Explanation of Anomalous Behavior in Models of Political Participation." American Political Science Review 99 (2): 201-13.

Hansen, John Mark. 1985. "The Political Economy of Group Membership." American Political Science Review 79 (1): 79-96.

Krasa, Stefan, and Mattias K. Polborn. 2009. "Is Mandatory Voting Better than Voluntary Voting?" Games and Economic Behavior 66 (1): 275-91.

Levine, David K., and Thomas R. Palfrey. 2007. "The Paradox of Voter Participation? A Laboratory Study." American Political Science Review 101 (1): 143-58.

Marwell, Gerald, and Pamela E. Oliver. 1991. "A Theory of the Critical Mass." In Disziplin und Kreativität, edited by Henrik Kreutz and Johann Bacher, 49-62. Berlin: Springer.

Myatt, David P. 2015. “A Theory of Voter Turnout.” http://dpmyatt.org/uploads/turnout-2015.pdf.

Myatt, David P., and Chris Wallace. 2008. "When Does One Bad Apple Spoil the Barrel? An Evolutionary Analysis of Collective Action." Review of Economic Studies 75 (2): 499-527.

Offerman, Theo, Joep Sonnemans, and Arthur Schram. 1996. "Value Orientations, Expectations and Voluntary Contributions in Public Goods." Economic Journal 106 (437): 817-45.

Olson, Mancur. 1965. The Logic of Collective Action: Public Goods and the Theory of Groups. Cambridge, MA: Harvard University Press.

Ordeshook, Peter C. 1986. "Basic Theory of Noncooperative Games." In Game Theory and Political Theory: An Introduction, 97-143. Cambridge, UK: Cambridge University Press.

-Osborne, Martin J., Jeffrey S. Rosenthal, and Matthew A. Turner. 2000. "Meetings with Costly Participation." American Economic Review 90 (4): 927-43.

Palfrey, Thomas R., and Howard Rosenthal. 1984. "Participation and the Provision of Discrete Public Goods: A Strategic Analysis.” Journal of Public Economics 24 (2): 171-93.

Palfrey, Thomas R., and Howard Rosenthal. 1988. "Private Incentives in Social Dilemmas: The Effects of Incomplete Information and Altruism." Journal of Public Economics 35 (2): 309-32.

Palfrey, Thomas, Howard Rosenthal, and Nilanjan Roy. 2017. "How Cheap Talk Enhances Efficiency in Threshold Public Goods Games." Games and Economic Behavior 101: 234-59.

Roberts, Russell D. 1984. "A Positive Model of Private Charity and Public Transfers." Journal of Political Economy 92 (1): 136-48. 
Saijo, Tatsuyoshi, and Takehiko Yamato. 1999. "A Voluntary Participation Game with a Non-excludable Public Good.” Journal of Economic Theory 84 (2): 227-42.

Sandler, Todd. 2015. "Collective Action: Fifty Years Later.” Public Choice 164 (1): 195-216.

Shadmehr, Mehdi. Forthcoming. "Protest Puzzles: Tullock's Paradox, Hong Kong Experiment, and the Strength of Weak States." Quarterly Journal of Political Science.

Shakiba, Mehdi. 2019. "Do Senators Take Strategic Advantage of Their Last Names?" https://drive. google.com/file/d/1ByKd57urIxJmOuaaT-7YBbMxwZv3DGsU/view.

Spenkuch, Jörg L., B. Pablo Montagnes, and Daniel B. Magleby. 2018. "Backward Induction in the Wild? Evidence from Sequential Voting in the US Senate." American Economic Review 108 (7): 1971-2013.

Spiller, Jörg, and Friedel Bolle. 2017. "Experimental Investigations of Binary Threshold Public Good Games.” https://ideas.repec.org/p/zbw/euvwdp/393.html.

Taylor, Curtis R., and Huseyin Yildirim. 2010. "Public Information and Electoral Bias." Games and Economic Behavior 68 (1): 353-75.

Taylor, Michael, and Hugh Ward. 1982. "Chickens, Whales, and Lumpy Goods: Alternative Models of Public-Goods Provision.” Political Studies 30 (3): 350-70.

Yildirim, Huseyin. 2006. "Getting the Ball Rolling: Voluntary Contributions to a Large-Scale Public Project." Journal of Public Economic Theory 8 (4): 503-28. 
